\title{
Stress-induced RNASET2 overexpression mediates melanocyte apoptosis via the TRAF2 pathway in vitro
}

\author{
Q Wang ${ }^{1,3}, \mathrm{M}$ Jiang ${ }^{1,3}, \mathrm{~J} \mathrm{Wu}{ }^{1}, \mathrm{Y} \mathrm{Ma}{ }^{1}, \mathrm{~T} \mathrm{Li}^{1}, \mathrm{Q}$ Chen ${ }^{1}, \mathrm{X}$ Zhang ${ }^{2}$ and L Xiang ${ }^{*, 1}$
}

The recent genome-wide association study identified a link between vitiligo and genetic variants in the ribonuclease T2 (RNASET2) gene; however, the functional roles of RNASET2 in vitiligo pathogenesis or in melanocyte apoptosis have yet to be determined. The current study was designed to investigate the vitiligo-related expression pattern of RNASET2 and its molecular function involving apoptosis-related signaling proteins and pathways. The results showed overexpression of RNASET2 in epidermis specimens from $\mathbf{4 0}$ vitiligo patients compared with that from matched healthy controls. In addition, in vitro analyses indicated that overexpression of RNASET2 was inducible in cultured primary human melanocytes and keratinocytes by stress conditions, that is, exposure to UV irradiation, hydrogen peroxide, and inflammatory factors, respectively, and led to increased cell apoptosis via the tumor necrosis factor receptor-associated factor 2 (TRAF2)-caspases pathway through the physical interaction of RNASET2 with TRAF2. Thus, RNASET2 may contribute to vitiligo pathogenesis by inhibiting TRAF2 expression and, as such, RNASET2 may represent a potential therapeutic target of vitiligo.

Cell Death and Disease (2014) 5, e1022; doi:10.1038/cddis.2013.539; published online 23 January 2014

Subject Category: Experimental Medicine

Vitiligo is classified as an idiopathic, acquired, circumscribed disease, and current global prevalence estimates range between 0.5 and $2 \%$. The primary physical symptom is patchy depigmentation of the skin and overlying hair, which manifests from a loss of functioning epidermal melanocytes. ${ }^{1,2}$ Studies of the pathogenic mechanisms of vitiligo have characterized the molecular disease process as multifactorial and complex, but have yet to identify the precise set of factors or their dynamic interactions that promote vitiligo development and progression. However, several theories have been put forth to explain vitiligo pathogenesis based on the histological, clinical, and etiological findings, the most popular of which involve autoimmunity, genetic factors, and oxidative stress. Although it is likely that the actual disease mechanism involves a complex interaction of multiple processes; for example, accumulated genetic and epigenetic changes may increase melanocytes' sensitivity to injury or destruction by excessive UV irradiation, hydrogen peroxide $\left(\mathrm{H}_{2} \mathrm{O}_{2}\right)$, and inflammatory factors (all of which are defined as vitiligo risk factors), it is also possible that perturbations in apoptosis signaling pathways contribute to the loss of melanocytes. ${ }^{3}$ In a recent genome-wide association study (GWAS) of generalized vitiligo in a Chinese Han population, the gene encoding ribonuclease T2 (RNASET2) was identified as a disease risk factor. ${ }^{4}$ The ribonucleases (RNases) are ubiquitous, conserved enzymes that function in a broad spectrum of normal physiological processes, such as host immune responses.
As such, perturbations in their expression profiles have been associated with various pathological conditions, including cancers and autoimmune diseases. Studies to determine the role of RNASET2 in cancers have elucidated its suppressive effects - independent of its nuclease activity - on cell growth and metastatic potential, both in vitro and in vivo. ${ }^{5-7}$ To date, RNASET2 is the only member of the Rh/T2/S family of acidic hydrolases identified in humans, ${ }^{8}$ but more recent studies of the RNASET2 ortholog in Saccharomyces cerevisiae, Rny1, have demonstrated a similar function, in that Rny1 can mediate inhibition of cell growth in response to oxidative stress conditions and that this activity is independent of the enzyme's catalytic activity. ${ }^{9}$

In silico analysis of the RNASET2 amino acid sequence identified a putative binding motif for the tumor necrosis factor receptor-associated factor 2 (TRAF2), ${ }^{10}$ which has recently been recognized as a threshold determinant factor of apoptosis through its ubiquitin ligase activities acting on caspase-8. ${ }^{11}$ This finding becomes particularly intriguing when considered in the context of the clinical and laboratory histology data of vitiligo-affected tissues that have demonstrated apoptosis, rather than cell death, is responsible for the melanocyte loss. ${ }^{12}$ Therefore, we postulated that the interaction of TRAF2 and RNASET2 may contribute to vitiligo pathogenesis in humans, possibly by perturbing the apoptosis mechanism in the involved cells (melanocytes and keratinocytes).

\footnotetext{
${ }^{1}$ Department of Dermatology, Huashan Hospital, Fudan University, Shanghai 200040, China and ${ }^{2}$ Institute of Dermatology, Anhui Medical University, Hefei, China *Corresponding author: L Xiang, Department of Dermatology, Huashan Hospital, Shanghai Medical College, Fudan University, 12 Wulumuqi Zhong Road, Shanghai 200040, China. Tel: + 86215288 9999; Fax: + 86216248 9191; E-mail: flora_xiang@vip.163.com

${ }^{3}$ These authors contributed equally to this work.

Keywords: stress; RNASET2; melanocyte; apoptosis; TRAF2

Abbreviations: RNASET2, ribonuclease T2; TRAF2, tumor necrosis factor receptor-associated factor 2; GWAS, genome-wide association study; HEM, primary human epidermal melanocyte; HEK, primary human epidermal keratinocyte; NB-UVB, narrow-band UVB; $\mathrm{H}_{2} \mathrm{O}_{2}$, hydrogen peroxide; LPS, lipopolysaccharide; $\mathrm{OE}$, overexpression; RNASET2-ci, catalytically inactive mutant of RNASET2; RNASET2-ti, RNASET2 lacking the TRAF2-binding domain; POMC, pro-opiomelanocortin; $\alpha$-MSH, $\alpha$-melanocyte stimulating hormone; MITF, microphthalmia-associated transcription factor; TYR, tyrosinase; TRP-1, TYR-related protein-1

Received 05.9.13; Received 28.11.13; accepted 02.12.13; Edited by A Stephanou
} 
Herein, we describe our study using in vitro approaches to determine the vitiligo-related expression pattern of RNASET2 using patient specimens and healthy tissues from matched control donors, and to uncover the molecular mechanism involving RNASET2 mediating the TRAF2-caspase signaling pathway of apoptosis using cultured primary human epidermal melanocytes (HEMs) and keratinocytes (HEKs).

\section{Results}

Vitiligo epidermis has significantly enhanced RNASET2 expression. To investigate the functional implication of the GWAS-detected RNASET2 risk factor in vitiligo pathogenesis, the differential expression of RNASET2 between lesion tissues from vitiligo patients $(n=40)$ and healthy tissues from donors $(n=40)$ was detected by quantitative reversetranscription PCR (qRT-PCR) and western blotting. Both the transcript (vitiligo versus control: $4.056 \pm 1.115$ versus $1.034 \pm 0.166 ; P<0.001)$ and protein expressions of RNASET2 were found to be significantly higher in the vitiligo epidermis specimens (Figures $1 \mathrm{a}$ and b). Immunohistochemistry analysis of the vitiligo epidermis specimens showed strong cytoplasmic staining for RNASET2, and the staining in healthy tissues was significantly lower (Figure 1c).

Stress conditions induce increased expression of RNASET2 in HEKs and HEMs. To investigate whether stress was a potential mechanism of the observed enhancement of RNASET2 expression in vitiligo epidermal lesions, the effects of UV irradiation, $\mathrm{H}_{2} \mathrm{O}_{2}$, and inflammatory factors on HEKs and HEMs were examined in vitro. All three of the stress conditions induced RNASET2 expression (of both transcript and protein) in a concentration-dependent manner (Figures 2 and 3). Immunofluorescence analysis also

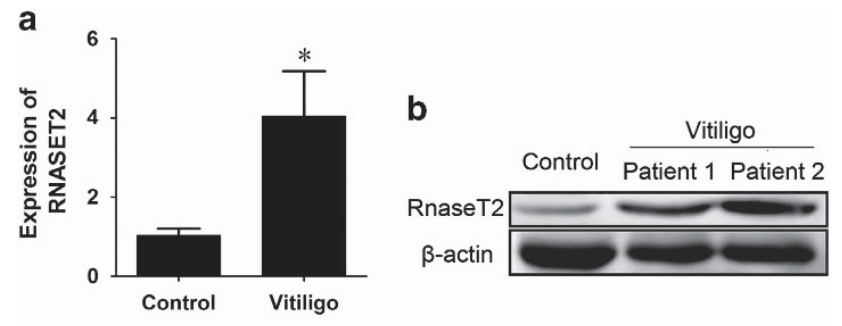

C

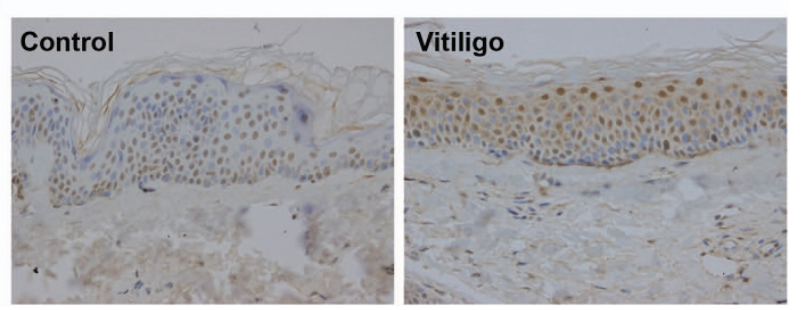

Figure 1 RNASET2 is overexpressed in clinical specimens of epithelial lesions from vitiligo patients. (a) RNASET2 mRNA expression in 40 vitiligo specimens and 40 healthy tissues detected by qRT-PCR and plotted as mean \pm S.D. from three independent experiments. ${ }^{*} P<0.01$. (b) RNASET2 protein expression in two representative vitiligo specimens and one healthy specimen detected by western blotting. (c) RNASET2 protein expression in representative healthy and vitiligo tissues detected by immunohistochemistry. Magnification: $\times 400$ showed concentration-dependent increases in the amount and dispersement of RNASET2 from the cytoplasm to the cell membrane and into the culture supernatant (indicating secreted into the extracellular space); in contrast, the control HEKs and HEMs under nonstress conditions showed the highest levels of RNASET2 in the cytoplasmic region nearest to the nucleus (Figures $2 \mathrm{~g}$ and $3 \mathrm{~g}$ ).

HEKs and HEMs overexpressing RNASET2 are less viable. To investigate whether the observed overexpression of RNASET2 in vitiligo epidermal lesions may have an effect on the survival the endothelial cells, the viability and apoptotic nature of HEKs and HEMs were assessed following lentivirus-mediated overexpression of RNASET2 (Figure 4). Both cell types showed reduced viability (Figures $4 \mathrm{e}, \mathrm{f}$ and $\mathrm{h}$ ) and increased apoptosis (Figures $4 \mathrm{~g}$ and i) when RNASET2 was overexpressed, as compared with the cells transduced with empty vector. Specifically, the percentage of cell apoptosis for VECTOR versus OE RNASET2 in HEK cells (4.167 \pm 0.330 versus $23.467 \pm 1.482 ; P=0.003)$ and HEM cells $(5.400 \pm 0.455$ versus $27.333 \pm 1.053 ; P=0.001)$ were with statistical significance. To prove that more an apoptosis induction instead of cell cycle was indeed relevant to RNASET2 overexpression, we carried out the cell cycle analyses of transfected HEKs and found that RNASET2 overexpression did not cause obvious disruption in cell cycle (data not shown). Moreover, we incubated the RNASET2overexpression HEK and HEM cells in the absence or presence of $10 \mu \mathrm{M} z V A D-f m k$, and the percentage of cell apoptosis for OE RNASET2 versus OE RNASET2-10 $\mu \mathrm{M}$ zVAD in HEK cells $(23.467 \pm 1.482$ versus $5.567 \pm 0.713$; $P=0.002)$ and HEM cells $(27.333 \pm 1.053$ versus $7.600 \pm 0.980 ; P=0.001)$ were with statistical significance (Figures $4 \mathrm{~g}$ and $\mathrm{i}$ ). The results of apoptosis analyses and cell cycle analyses proved that RNASET2 overexpression was indeed relevant to cell apoptosis.

HEKs and HEMs overexpressing RNASET2 are hypersensitive to oxidative stress. Considering that overexpression of the yeast RNase RNY1 causes hypersensitivity to $\mathrm{H}_{2} \mathrm{O}_{2}$-induced oxidative stress, ${ }^{9}$ we investigated whether RNASET2 has an analogous biological function in vitiligo pathogenesis. First, we conducted preliminary doseresponse $(0-100 \mu \mathrm{M})$ and kinetic (1-24h) experiments, and gained the determination of the optimal conditions for oxidative stress, that is, HEK: $5 \mu \mathrm{M} \mathrm{H}_{2} \mathrm{O}_{2}$ for $24 \mathrm{~h}$ and HEM: $0.5 \mu \mathrm{M} \mathrm{H}_{2} \mathrm{O}_{2}$ for $24 \mathrm{~h}$ (Figures $5 \mathrm{a}$ and b). Indeed, the lentivirus-mediated RNASET2 overexpression caused hypersensitivity of $\mathrm{HEKs}$ and $\mathrm{HEMs}$ to $\mathrm{H}_{2} \mathrm{O}_{2}$-induced oxidative stress, as evidenced by the higher rate of net cell apoptosis (Figures 5c and d) and characteristic morphological changes (Figures $5 e$ and f) as compared with the cells transduced with empty vector.

RNASET2-overexpressing HEKs and HEMs have reduced melanin synthesis. To further investigate the functional role of RNASET2 in vitiligo pathogenesis, several of the catalytic and signaling factors previously identified as related to the disease process were investigated in the HEKs and HEMs with lentivirus-mediated RNASET2 overexpression. 


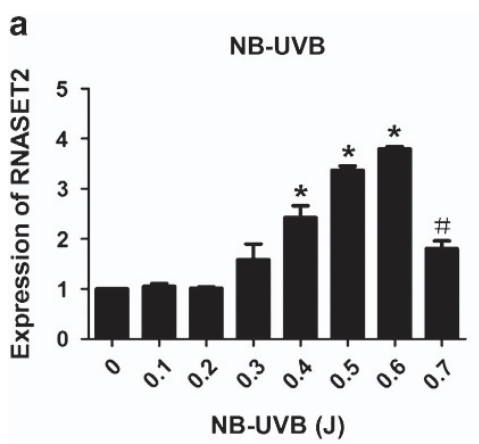

b

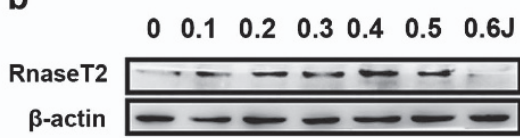

C

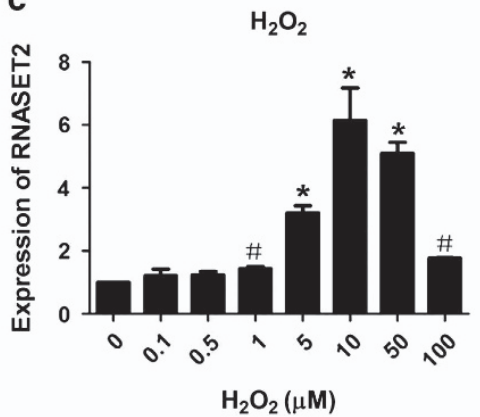

d e

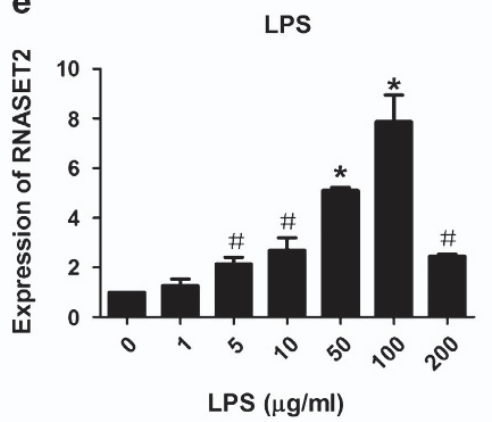

f
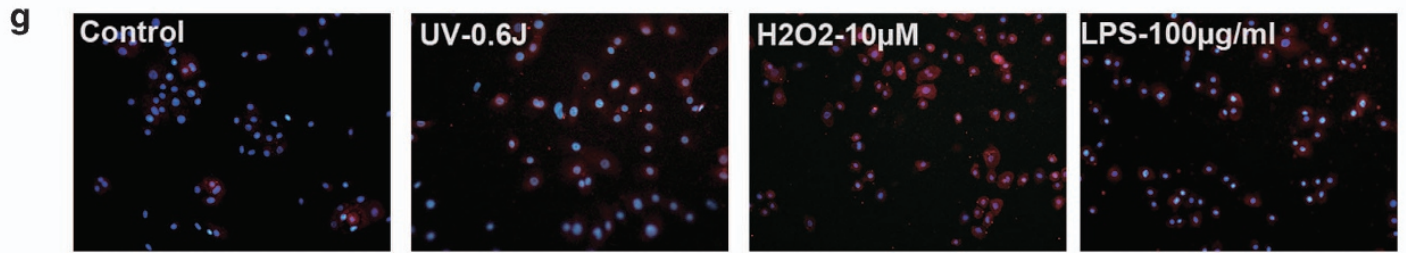

Figure 2 Stress-induced RNASET2 expression in HEKs. (a and $\mathbf{b}$ ) Excessive UV irradiation. (c and $\mathbf{d}) \mathrm{H}_{2} \mathrm{O}_{2}$. (e and f) LPS. (a, $\mathbf{c}$, and $\mathbf{e}$ ) Plots of $\beta$-actin-normalized RNASET2 mRNA expression detected by qRT-PCR after $24 \mathrm{~h}$ under stress conditions. Data are expressed as the mean \pm S.D. of values from three independent experiments. ${ }^{\#} P<0.05,{ }^{*} P<0.01$ versus control group, by one-way ANOVA with Dunnett's multiple comparison test. (b, $\mathbf{d}$, and $\left.\mathbf{f}\right)$ Western blotting detection of RNASET2 protein expression after $48 \mathrm{~h}$ under stress conditions. (g) Indirect immunofluorescence detection of RNASET2 protein expression in HEKs after $48 \mathrm{~h}$ under stress conditions. Magnification: $\times 200$. Western blotting and immunofluorescence experiments were repeated at least three times and representative results are shown

The HEKs overexpressing RNASET2 showed significantly decreased pro-opiomelanocortin (POMC) expression at both the transcript (Figure 6a) and protein (Figure 6b) levels. The HEMs overexpressing RNASET2 showed reduced tyrosinase (TYR) activity (Figure 6c) and melanin synthesis (Figure 6d).

To investigate the effects of RNASET2 overexpression on melanin synthesis in HEMs, the expression levels of its key factors, microphthalmia-associated transcription factor (MITF), TYR, and TYR-related protein-1 (TRP-1), were detected. Both the transcript (Figure 6e) and protein (Figure 6f) levels of all three factors were significantly downregulated in RNASET2-overexpressing HEMs, as compared with control HEMs transfected with empty vector. These results suggest that RNASET2 overexpression might directly decrease melanogenesis of HEMs in vitro.

RNASET2 contributes to cell death through its TRAF2binding motif, but independent of its catalytic activity. To investigate the mechanism by which RNASET2 mediates cell death, the effects of disrupting two of its function-related components, the catalytic site and the predicted TRAF2binding motif, were examined. The loss of HEK and HEM cell viability and increased apoptosis observed on overexpression of RNASET2 were similar to that observed on overexpression of a catalytically inactive version of RNASET2 (Figure 7). Specifically, the percentage of cell apoptosis for VECTOR versus OE of catalytically in active mutant of RNASET2 (RNASET2-ci) in HEK cells $(4.533 \pm 0.351$ versus $22.300 \pm 1.374 ; P=0.001)$ and HEM cells $(5.6 \pm 0.748$ versus $26.8333 \pm 1.144 ; P=0.001)$ were with statistical significance (Figures $7 g$ and h). However, overexpression of a TRAF2-binding site mutant version of RNASET2 did not produce the same reduced cell viability (Figures 7e and $\mathrm{f}$ ) and high levels of apoptosis both in HEK cells (OE RNASET2-ti versus OE RNASET2: $6.367 \pm 0.903$ versus $21.667 \pm 0.939 ; \quad P=0.002$ ) and HEM cells (OE RNASET2-ti versus OE RNASET2: $7.367 \pm 1.066$ versus $27.267 \pm 1.087 ; P=0.004$; Figures $7 \mathrm{~g}$ and $\mathrm{h}$ ) as the wild-type RNASET2 overexpression, suggesting a potential pathologically relevant role for RNASET2-TRAF2 interaction in vitiligo. To further prove that RNASET2-overexpressing cells are hypersensitive to oxidative stress through its TRAF2binding motif but independent of its catalytic activity, we incubated transfected HEK and HEM cells (neo, RNASET2 transfected or RNASET2 mutants transfected) with optimal $\mathrm{H}_{2} \mathrm{O}_{2}$ concentration as indicated for $24 \mathrm{~h}$, and found that the RNASET2-ti mutant protected HEK and HEM cells from apoptosis triggered by $\mathrm{H}_{2} \mathrm{O}_{2}$, whereas RNASET2-ci mutant did not. Specifically, the levels of cell apoptosis after oxidative stress for OE of RNASET2-ti versus OE of RNASET2 in HEK cells $(14.667 \pm 0.710$ versus $40.667 \pm 1.856 ; P=0.001)$ and HEM cells $(18.533 \pm 0.713$ versus $54.900 \pm 1.445 ; \quad P=0.002)$ were with statistical significance (Figures $7 \mathrm{~g}$ and $\mathrm{h}$ ).

RNASET2 promotes apoptosis via the TRAF2-caspase pathway. The RNASET2-TRAF2 physical interaction was confirmed by immunoprecipitation of a FLAG-tagged wildtype RNASET2 by anti-TRAF2 monoclonal antibody 

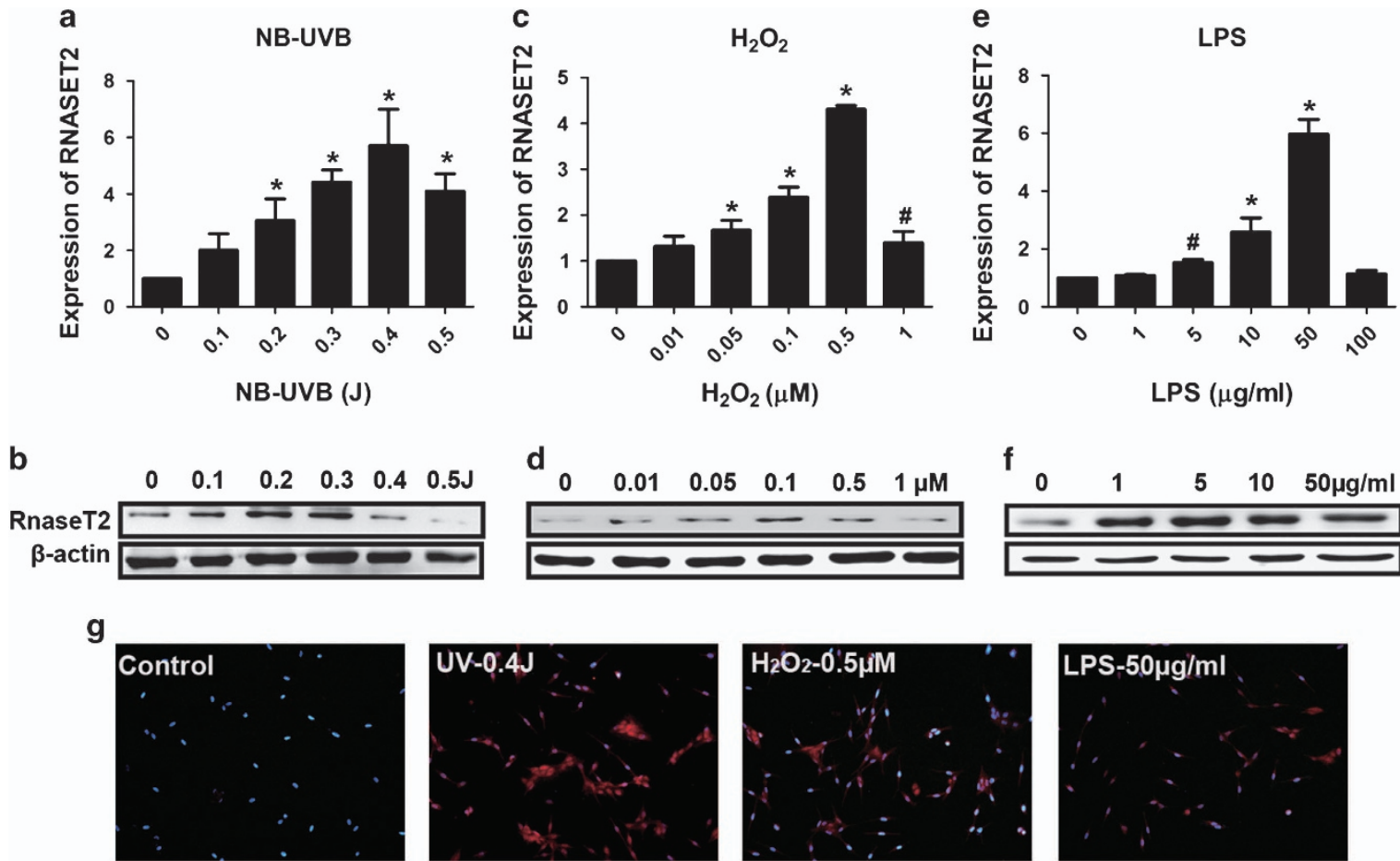

Figure 3 Stress-induced RNASET2 expression in HEMs. (a and $\mathbf{b}$ ) Excessive UV irradiation. (c and $\mathbf{d}) \mathrm{H}_{2} \mathrm{O}_{2}$. (e and $\mathbf{f}$ ) LPS. (a, c, and e) Plots of $\beta$-actin-normalized RNASET2 mRNA expression detected by qRT-PCR after $24 \mathrm{~h}$ under stress conditions. Data are expressed as the mean \pm S.D. of values from three independent experiments. ${ }^{\#} P<0.05$, ${ }^{*} P<0.01$ versus control group, by one-way ANOVA with Dunnett's multiple comparison test. (b, $\mathbf{d}$, and $\mathbf{f}$ ) Western blotting detection of RNASET2 protein expression after $48 \mathrm{~h}$ under stress conditions. (g) Indirect immunofluorescence detection of RNASET2 protein expression in HEKs after $48 \mathrm{~h}$ under stress conditions. Magnification: $\times 200$. Western blot and immunofluorescence experiments were repeated at least three times and representative results are shown

(Figure 8a). Next, to begin to explore the potential mechanism of the RNASET2-TRAF2 interaction, particularly in relation to effects on cell apoptosis, western blot analysis of differential expression of TRAF2, caspase-8, and caspase-3 were examined following RNASET2 overexpression. Lentivirus-induced RNASET2 overexpression in HEMs was found to be accompanied by decreased expression of TRAF2 and increased proteolytic activation of both caspase-8 and caspase-3 (Figures 8b and c).

\section{Discussion}

Vitiligo manifests on progressive destruction of melanocytes and has susceptibility components in both genetics and environmental exposures (i.e., UV, $\mathrm{H}_{2} \mathrm{O}_{2}$, and lipopolysaccharide (LPS)). Despite having identified various risk factors of vitiligo, the molecular mechanisms of its complex underlying pathogenesis remain largely unknown. The current study of RNASET2 provides novel insights into the mechanisms related to cell death and apoptosis of epithelial cells in affected tissues.

The mammalian RNases comprise a heterogeneous group of regulatory enzymes involved in a wide array of normal biological processes, including gene expression and cell differentiation. RNASET2 is a relatively newly recognized member of the Rh/T2/S family of RNases; as such, the data presented herein not only reveal a novel pathogenic network of vitiligo but also advance our understanding of RNASET2's general biological properties.
In particular, the current study provided clinically relevant evidence of RNASET2 overexpression in human vitiligo lesions, as compared with healthy tissue, and found that environmental stress conditions associated with vitiligo can stimulate RNASET2 expression in cultured human primary melanocytes and keratinocytes. Notably, these in vitro stress conditions appeared to also stimulate a dynamic movement of RNASET2 from the perinuclear region to the cell membrane and out to the extracellular space (data not shown); these results are preliminary and the ongoing detailed investigations are expected to confirm the extent to which this dynamic profile parallels that previously demonstrated for the yeast ortholog of RNASET2, Rny1p. ${ }^{8}$ Recent study demonstrated that overexpression of human RNASET2 in yeast results in tRNA and rRNA cleavage, and a growth defect. ${ }^{9}$ As tRNA and rRNA have decisive roles in protein synthesis process, tRNA and rRNA cleavage due to RNASET2 overexpression might lead to protein synthesis disorders. This might be the reason for the lack of correlation between RNASET2 mRNA and protein expression at $0.6 \mathrm{~J}$ narrow-band UVB (NB-UVB) radiation in HEK cells (Figure $2 \mathrm{a}$ ) and at 0.4 and $0.5 \mathrm{~J}$ NB-UVB radiation in HEM cells (Figure $3 b$ ), and at 100 and $200 \mu \mathrm{g} / \mathrm{ml}$ LPS in HEK cells (Figure $2 \mathrm{e}$ ).

Rny1p has been extensively studied and has been characterized as an intracellular circuit switch capable of controlling cell fate, irrespective of its cytosolic RNA cleavage ability. ${ }^{9,13}$ The previous study that showed oxidative stressinduced translocation of Rny1p from the vacuole into the cytosol also showed that this dynamic process leads to the 
a
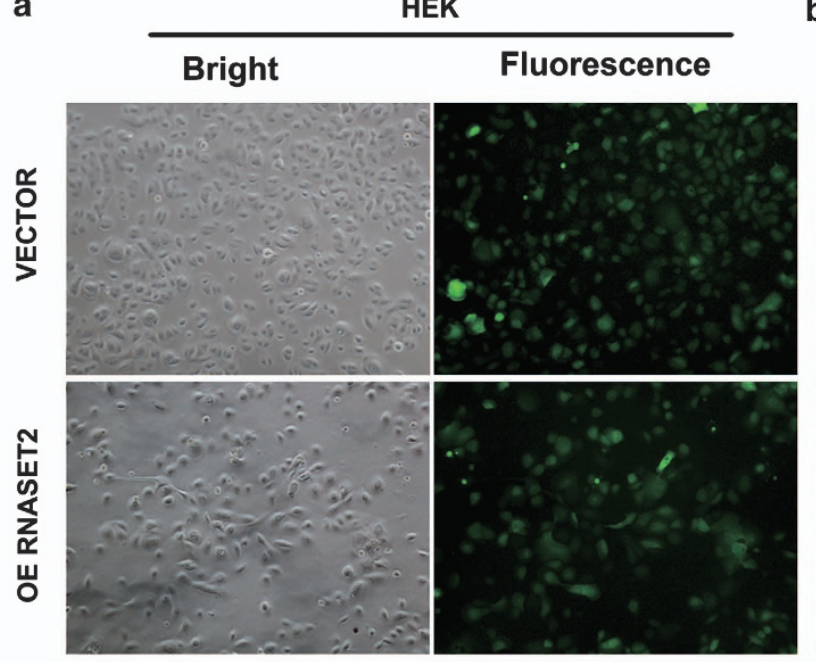

b

HEM

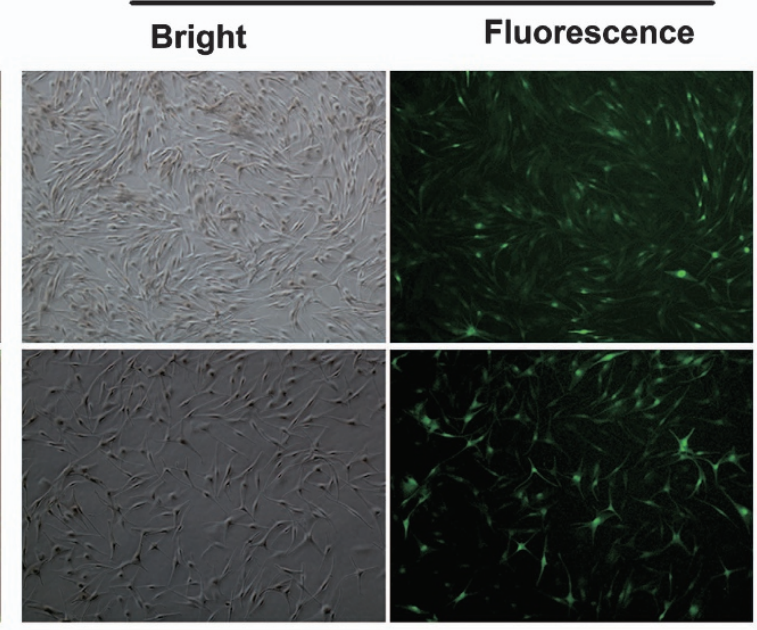

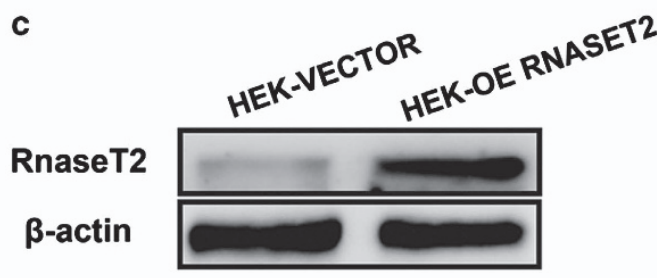
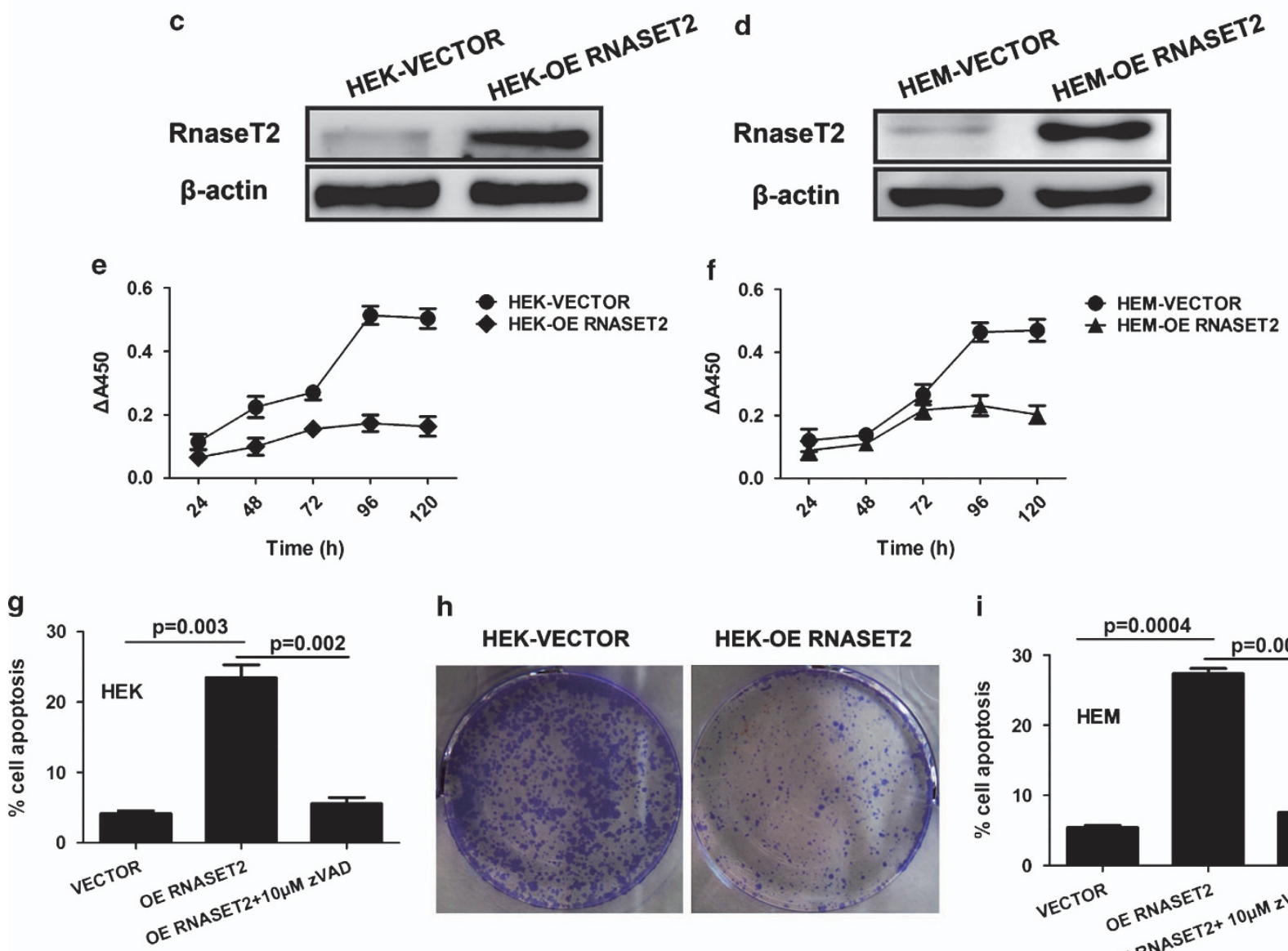

h
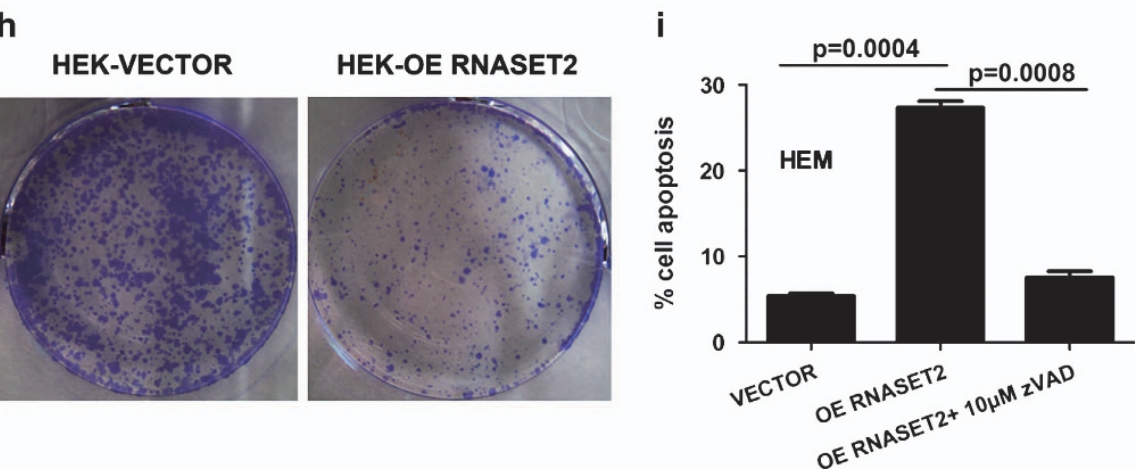

Figure 4 Lentivirus-mediated RNASET2 overexpression perturbs growth of HEKs and HEMs in vitro. At $72 \mathrm{~h}$ post transfection, (a and $\mathbf{b})$ image of HEKs (magnification: $\times 100)$ and HEMs $(\times 200)$ transfected with empty vector $($ VECTOR) or wild-type RNASET2-overexpressing vector (OE RNASET2), and (c and d) overexpression of RNASET2 in HEKs and HEMs confirmed by western blotting. Transfected cells were collected and seeded for further assays. (e and f) Plots of growth curves over time as indicated for HEKs and HEMs. (g and i) Analyses of HEKs and HEMs undergoing apoptosis in the absence or presence of $10 \mu \mathrm{M} \mathrm{zVAD}$-fmk for $24 \mathrm{~h}$ at day 3 after seeding. (e$\mathrm{g}$ and i) Data are expressed as the mean \pm S.D. of values from three independent experiments. (h) Colony-forming assay of HEKs was conducted at day 14 after seeding. Experiments were repeated at least three times and representative results are shown

activation of a cell apoptosis signaling pathway, independent of the enzyme's catalytic activity. ${ }^{9}$ This latter finding was also confirmed in an in vivo tumor model. ${ }^{14}$ In the current study,
RNASET2 was shown to induce apoptosis of human primary melanocytes and keratinocytes and to cause hypersensitivity to oxidative stress, irrespective of the enzyme's catalytic activity. 
a

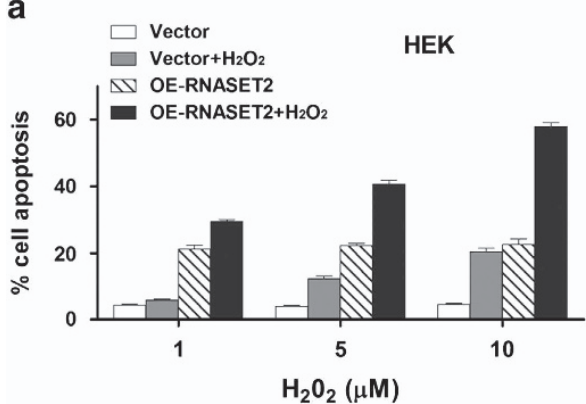

c
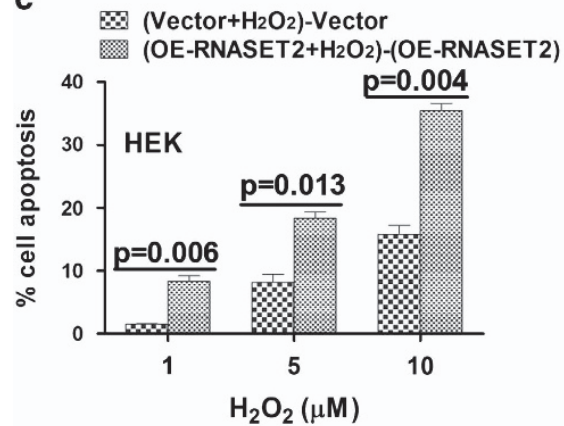

e

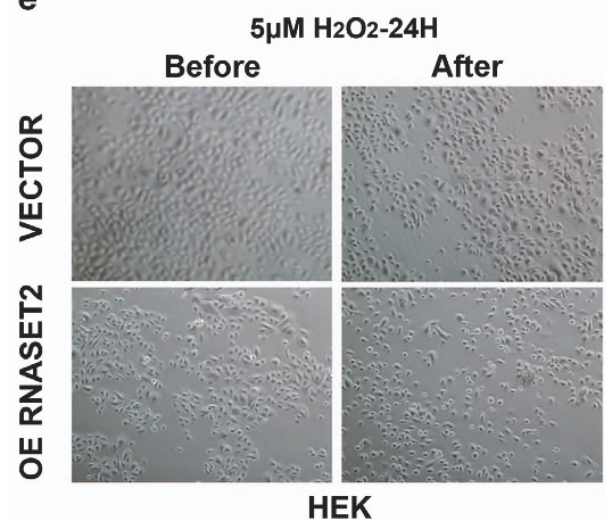

b

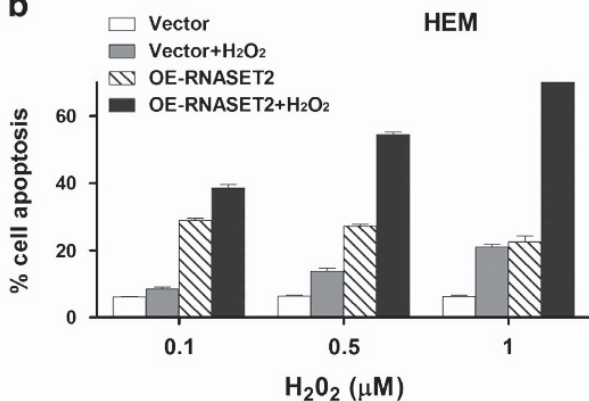

d

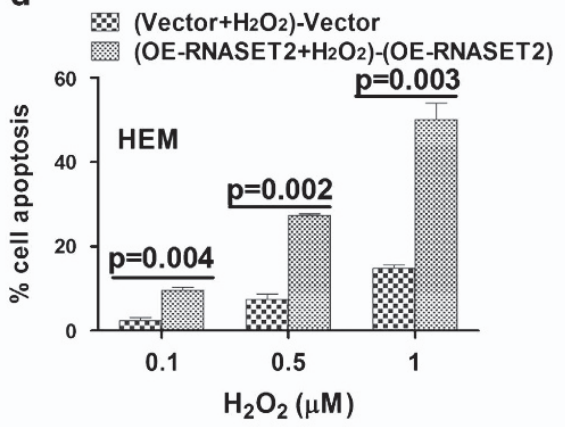

f

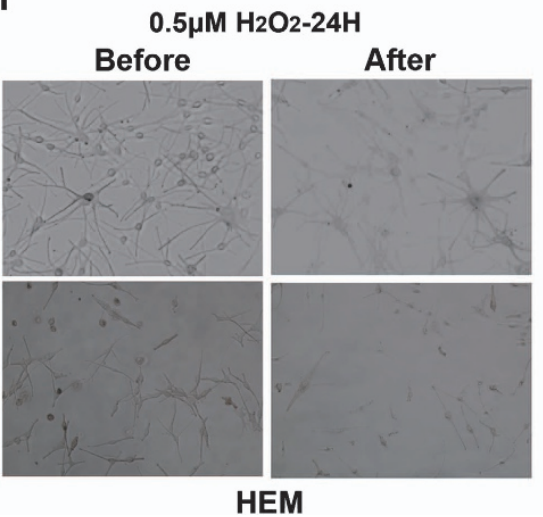

Figure 5 HEKs and HEMs overexpressing RNASET2 are hypersensitive to oxidative stress. Transfected cells were collected and seeded for further assays. All the experiments were conducted at day 3 after seeding. (a and b) Analyses of apoptosis with or without different $\mathrm{H}_{2} \mathrm{O}_{2}$ concentration for $24 \mathrm{~h}$ in $\mathrm{HEKs}(1,5$, and $10 \mu \mathrm{M})$ and $\mathrm{HEMs}$ $(0.1,0.5$, and $1 \mu \mathrm{M})$. (c and $\mathbf{d})$ Comparison of net cell apoptosis of $\mathbf{a}$ and $\mathbf{b}$ induced by $\mathrm{H}_{2} \mathrm{O}_{2}$ in $\mathrm{HEKs} / \mathrm{HEMs}$-VECTOR and HEKs/HEMs-OE RNASET2. Paired $t$-test was used to determine significance of between-group differences. (e and f) Changes in cell morphology before and during oxidative stress. HEKs-VECTOR and HEKs-OE RNASET2 were observed after a 24-h exposure to $5 \mu \mathrm{M} \mathrm{H}_{2} \mathrm{O}_{2}$ (left), whereas HEMs-VECTOR and HEMs-OE RNASET2 were observed after a 24-h exposure to $0.5 \mu \mathrm{M} \mathrm{H} \mathrm{H}_{2}$ (right). Experiments were repeated at least three times; representative examples are shown

These findings also agree with the biological properties demonstrated for other T2 RNase family members. ${ }^{15}$

The POMC-derived $\alpha$-melanocyte stimulating hormone $(\alpha-\mathrm{MSH})$ is expressed in the central nervous system and peripheral tissues such as the skin, with its primary skin expression in keratinocytes. ${ }^{16}$ Accordingly, robust functions of $\alpha-\mathrm{MSH}$ have been defined in the molecular processes of skin pigmentation, apoptosis, inflammation, and UV-induced immune suppression. ${ }^{17,18}$ Furthermore, $\alpha$-MSH stimulation of its cognate receptor on melanocytes, melanocortin 1 receptor, was shown to result in a G-protein-coupled induction of CAMP, which in turn drives the CRE-MITF pathway and promotes cell proliferation and melanin synthesis. ${ }^{19}$ Thus, the significantly decreased POMC expression observed in HEKs overexpressing RNASET2 of the current study may represent a direct (or indirect) link to poor melanocyte proliferation and suppressed melanin synthesis. MITF is considered a master regulatory transcription factor of melanocytes, directly regulating melanocyte-specific genes such as Tyr and Trp-1 (which are also related to melanin synthesis), ${ }^{20}$ its broadrange transcriptional effects have implications for cell survival, cell cycle progression, migration, and differentiation. ${ }^{21}$ Thus, the significantly decreased expression of Mitf, Tyr, and Trp-1 observed in HEMs overexpressing RNASET2 of the current study may represent a direct link to RNASET2 inhibition of melanogenesis (Figure 8d).

The molecular mechanisms RNASET2 overexpressioninduced apoptosis is another unknown feature of this enzyme. 
a

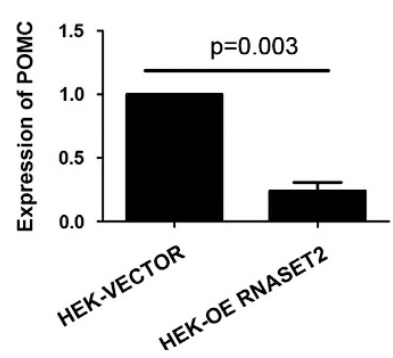

b

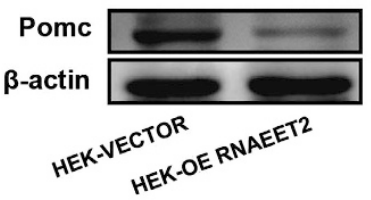

C

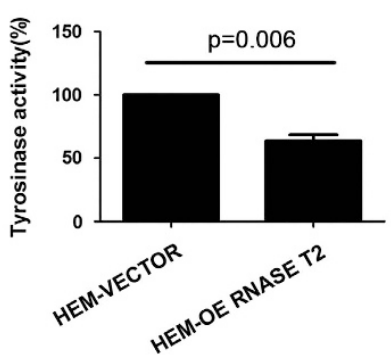

d

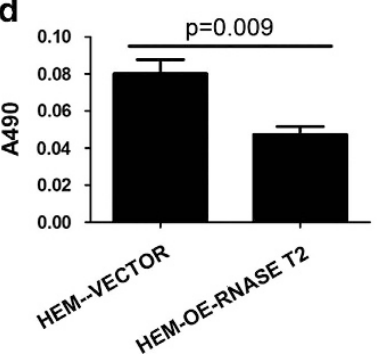

e
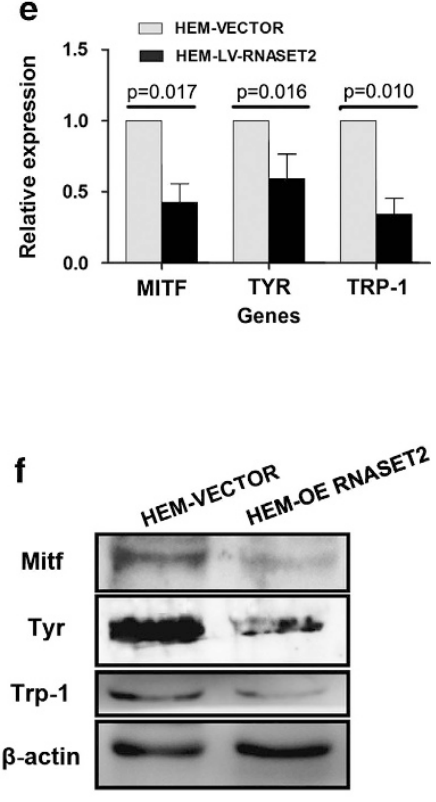

Figure 6 Overexpression of RNASET2 in HEKs and HEMs leads to reduced melanin synthesis. Transfected cells were collected and seeded for further assays. All the experiments were conducted at day 3 after seeding. (a and $\mathbf{b}$ ) In HEKs, OE RNASET2 significantly decreased the expression of POMC as detected by (a) qRT-PCR and (b) western blotting. (c-f) In HEMs, OE of RNASET2 significantly decreased melanin synthesis, as shown by (c) TYR activity assay and (d) melanin quantification. (e and f) In HEMs, OE of RNASET2 significantly decreased the expression of MITF, TYR, and TRP-1 as detected by (e) qRT-PCR and (f) western blotting. Quantitative data are presented as mean \pm S.D. from three independent experiments. Qualitative experiments were repeated at least three times and representative results are shown

A previous analysis of the RNaseT2 structure to provide insight into its biological roles by identifying putative functional sites found a conserved TRAF2-binding motif. ${ }^{10}$ As we all know, the apoptosis-related mechanisms of caspase activation are well defined, yet the mechanisms and functional roles of the countered inactivation modes remain unclear. Recent study reported that TRAF2 interacts with caspase-8 at the death-inducing signaling complex. TRAF2 has a critical function in regulation of apoptosis by directing RINGdependent, K48-linked polyubiquitination of caspase-8, effectively marking the protease for proteasomal degradation on autoprocessing and cytoplasmic translocation. Thus, TRAF2 depletion lowers the signal threshold for apoptosis. ${ }^{11}$ In the current study, we observed TRAF2 downregulation accompanying with RNASET2 overexpression. As TRAF2 set a critical barrier for cell apoptosis commitment by tagging activated caspase-8 with a K48-ubiquitin shutoff timer, TRAF2 downmodulation could lower the signal threshold for apoptosis, and then activation (via cleavage) of caspase-3, a downstream effector of caspase-8, was increased in cells with RNASET2 overexpression (Figure $8 \mathrm{~d}$ ). It is possible then that RNASET2 overexpression might promote apoptosis via the TRAF2-caspase pathway, and this process would be independent of its catalytic activity. Strikingly, the results in the current study demonstrated that when the TRAF2-binding site in RNASET2 was mutated, the promoting effects on apoptosis were disrupted. We have also conducted RNASET2-ticoimmunoprecipitation and found that RNASET2-ti do indeed fail to immunoprecipitate TRAF2 or obviously weaken the interaction compared with the wide-type RNASET2 (data not shown). The results proved that the RNASET2-ti mutant cannot support TRAF2 binding, thus further confirming that
RNASET2 and TRAF2 do indeed interact. These findings served to verify physiological relevance for the predicted TRAF2 site. To investigate a detailed downstream mechanism, we have designed a set of experiments for the next stage. We will re-confirm the specific interaction between RNASET2 and TRAF2 critical for cell death in a more physiologic system (zebrafish model) and explore the effects of RNASET2 on TRAF2 (transcriptional, translational, or posttranslational?). As importantly, the co-immunoprecipitation of RNASET2 and TRAF2 in this study represents, to the best of our knowledge, the first reported evidence of a relationship between RNASET2 and TRAF2 in human cells.

Furthermore, autoimmunity is also involved in the pathogenesis of vitiliogo. Recent studies also indicated that omega1, an RNase T2 family member secreted from the eggs of Schistosoma mansoni, contributes to Th2 polarization of $\mathrm{CD}^{+} \mathrm{T}$ cells by priming human dendritic cells, ${ }^{22,23}$ hinting that human RNASET2 might be involved in immune response, and therefore underlie the pathogenesis of vitiligo.

In summary, the study presented herein demonstrates that RNASET2 protein overexpression is associated with vitiligo and that the underlying pathogenic mechanism may involve RNASET2 stimulation of cell apoptosis and hypersensitization to stress via the TRAF2-caspase pathway. The molecular mechanisms of RNASET2 uncovered by this study may represent manipulable features by which the clinical management of vitiligo patients may be improved.

\section{Materials and Methods}

Patients and tissue specimens. Split-thickness grafting was used to obtain stable stage vitiligo-affected skin tissue specimens from 40 patients (female/male: 22/18; mean age in years: $28.2 \pm 10.3$ ) at the Huashan Hospital 

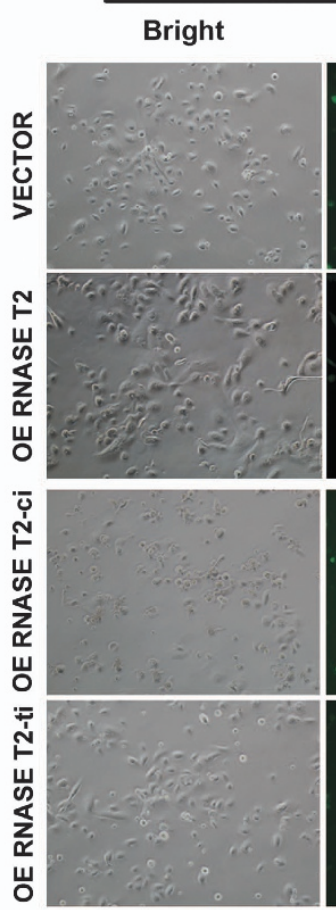

HEK

\section{Fluorescence}
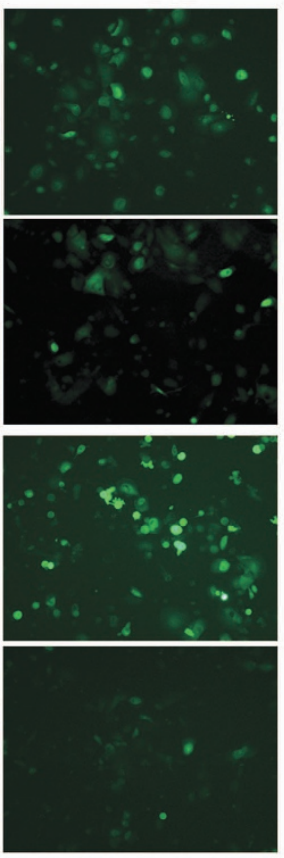

b

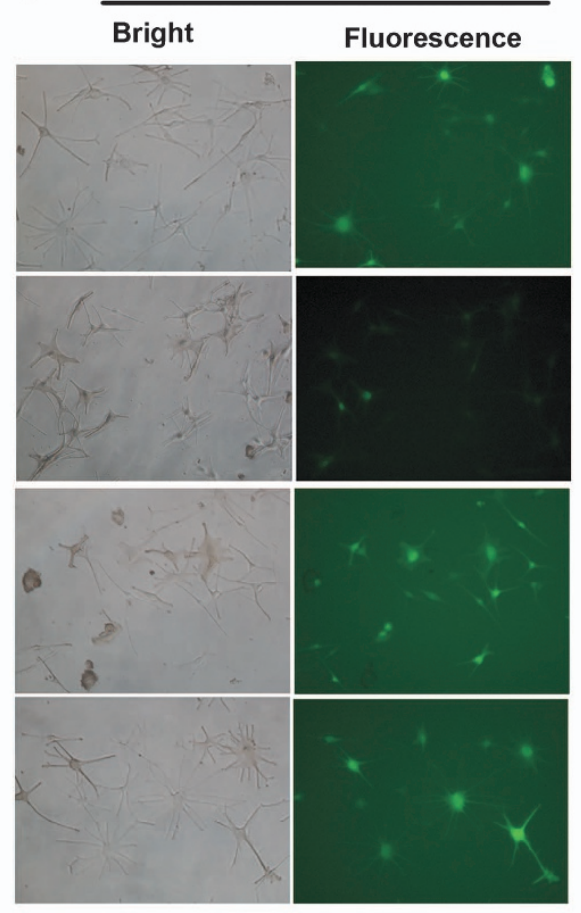

c
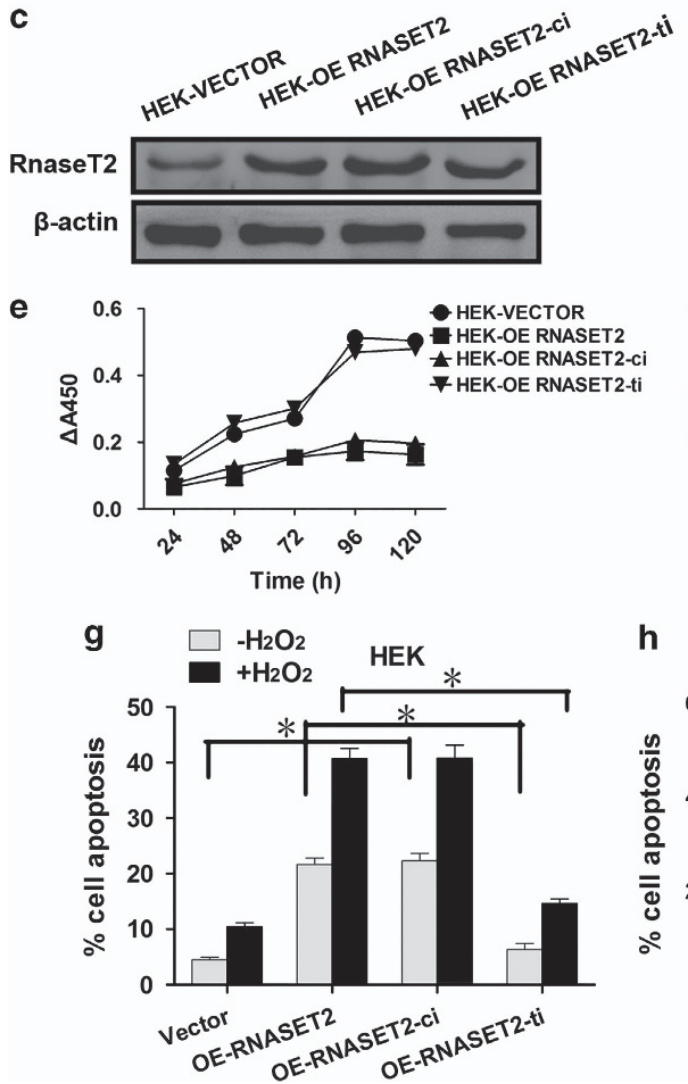

d
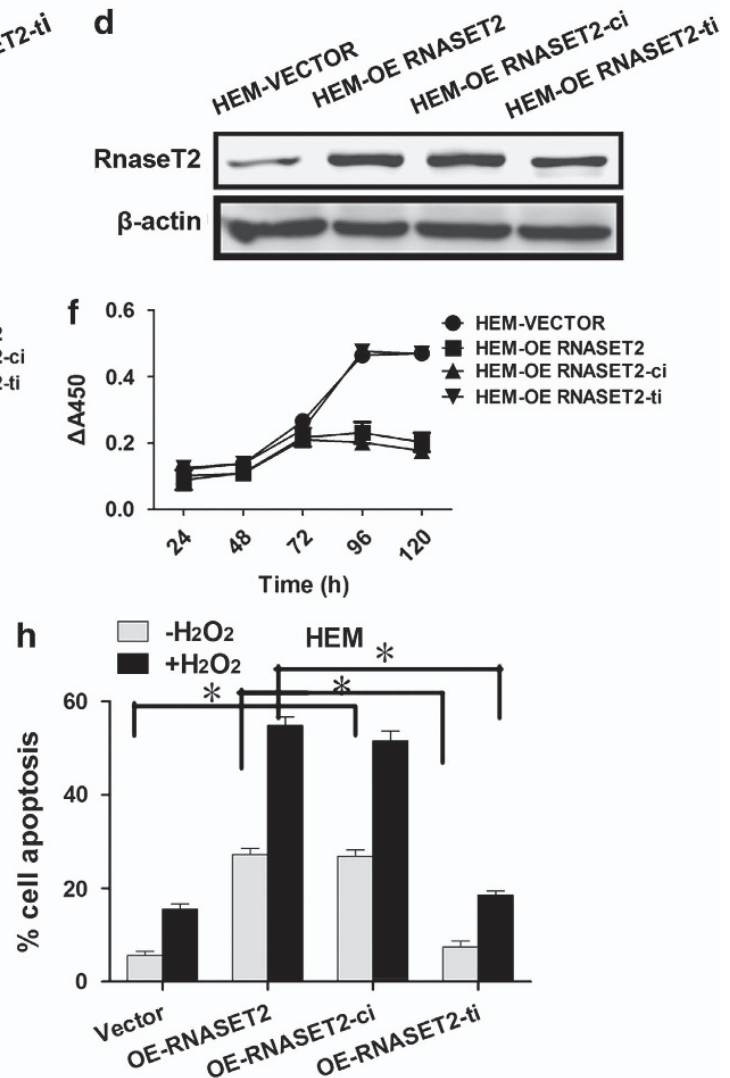

Figure 7 Contribution of the catalytic activity and TRAF2-binding site to cell growth effects mediated by RNASET2 overexpression in HEKs and HEMs. At $72 \mathrm{~h}$ post transfection, $(\mathbf{a}$ and $\mathbf{b})$ image of HEKs (magnification: $\times 100)$ and HEMs $(\times 200)$ transfected with empty vector (VECTOR), wild-type RNASET2-overexpressing vector (OE RNASET2), catalytically inactive RNASET2-overexpressing vector (OE RNASET2-ci), or TRAF2-binding site mutant RNASET2-overexpressing vector (OE RNASET2-ti), and ( $\mathbf{c}$ and $\mathbf{d}$ ) RNASET2 overexpression in HEKs and HEMs confirmed by western blotting. Transfected cells were collected and seeded for further assays. (e and $\mathbf{f}$ ) Plots of growth curves over time as indicated for HEKs and HEMs. ( $\mathbf{g}$ and $\mathbf{h}$ ) Apoptosis assays of HEKs with or without exposure to $5 \mu \mathrm{M} \mathrm{H}_{2} \mathrm{O}_{2}$ (left) and $\mathrm{HEMs}$ with or without exposure to $0.5 \mu \mathrm{M} \mathrm{H}_{2} \mathrm{O}_{2}$ (right) for $24 \mathrm{~h}$ at day 3 after seeding. Data are expressed as the mean \pm S.D. of values from three independent experiments. ${ }^{*} P<0.01$ 

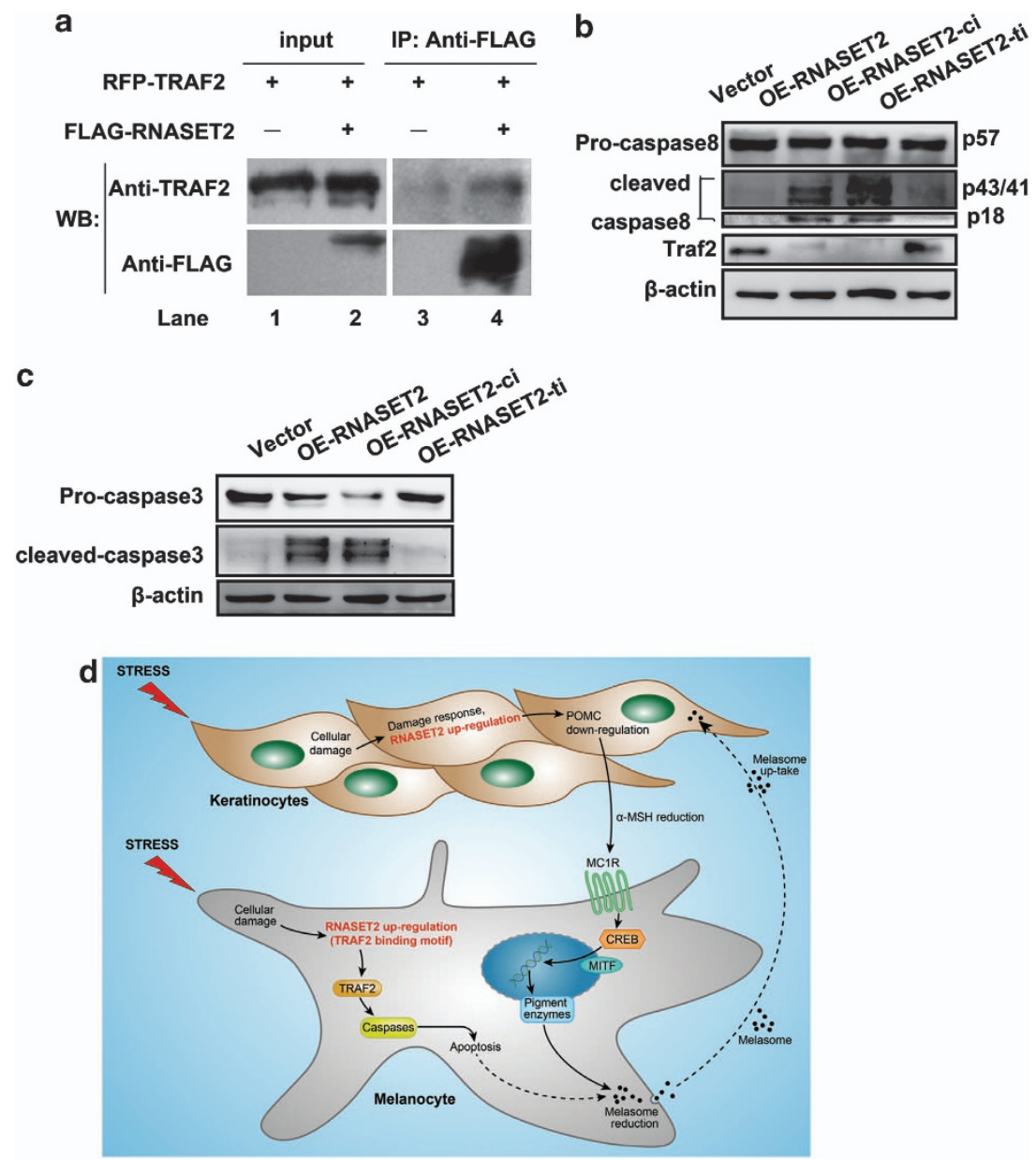

Figure 8 RNASET2 promotes apoptosis via the TRAF2-caspase pathway. (a) RNASET2 immunoprecipitation with TRAF2. pmRFP-TRAF2 was transfected into H1299 cells along with GV287-RNASET2-FLAG (lanes 2 and 4) or alone (lanes 1 and 3). Western blotting membrane probed with an antibody against TRAF2 (upper) and against FLAG (lower) is shown. (b) Western blotting detection of TRAF2 and caspase-8 expression in HEMs. OE of RNASET2 and OE of RNASET2-ci produced proteolytic activation of caspase-8 and low TRAF2 expression compared with VECTOR and OE RNASET2-ti. (c) Western blotting detection of caspase-3 expression. (d) Hypothesized contribution of RNASET2 protein overexpression in the pathogenesis of vitiligo. RNASET2 may promote cell apoptosis and hypersensitization to stress via the TRAF2-caspase pathway

affiliated to Fudan University, China. Non-vitiligo tissue specimens were obtained from healthy volunteer donors $(20 / 20 ; 30.1 \pm 11.2)$ for use as controls. All specimens were stored at $-80^{\circ} \mathrm{C}$ until processing for extraction of RNA and protein.

The study was carried out with pre-approval from the Independent Ethics Committee of Huashan Hospital.

Primary HEM and HEK cultures. Primary HEM and HEK cultures were performed as described previously, ${ }^{24}$ and were cultured under standard conditions in either M254-defined phorbol-12-myristate-13-acetate-free medium supplemented with human melanocyte growth factors (Invitrogen, Carlsbad, CA, USA) or keratinocyte-defined serum-free medium supplemented with human keratinocyte growth factors (Invitrogen). For the stress condition cultures, HEMs and HEKs were used at passage 2 or 3 , and treated with different concentrations of risk factors, such as excessive NB-UVB exposure (Sigma-Aldrich, St. Louis, MO, USA), $\mathrm{H}_{2} \mathrm{O}_{2}$ purchased from Sangon Biotech (Shanghai, China) and LPS purchased from Sigma-Aldrich.

Real-time quantitative reverse-transcription -PCR. Cells were lysed and total RNA was extracted from the tissue specimens and primary cells by the SV Total RNA Isolation System (Promega, Madison, WI, USA), and used to generate cDNA with the PrimeScript RT Reagent Kit (Takara, Ōtsu, Shiga, Japan). The cDNA was applied as template for qRT-PCR, which was carried out in a
Real-Time PCR 7500 System (Applied Biosystems, Invitrogen) with SYBR Premix ExTaq (Takara) in a 40-cycle PCR. The denaturing, annealing, and extension conditions of each PCR cycle were $95^{\circ} \mathrm{C}$ for $30 \mathrm{~s}, 95^{\circ} \mathrm{C}$ for $5 \mathrm{~s}$, and $60^{\circ} \mathrm{C}$ for $34 \mathrm{~s}$, respectively. The following were gene-specific forward and reverse primer pairs: RNASET2: $5^{\prime}$-GAGTGATACCCAAAATCCAGT-3' and $5^{\prime}$-GCTTAGTGAGGCAC AGTTCT-3'; MITF: 5'-ACAACCTGATTGAACGAAG- $3^{\prime}$ and $5^{\prime}$-AATCTGGAGAGC AGAGACC-3'; TYR: $5^{\prime}$-TTGGCAGATTGTCTGTAGCC-3' and $5^{\prime}$-AGGCATTGTG CATGCTGCTT-3'; TRP-1: $5^{\prime}$-ACTTTGTAACAGCACCGAGGAT-3' and $5^{\prime}$-ATGT CCAATAGGGGCATTTTC- $3^{\prime}$; POMC: $5^{\prime}$-CTACGGCGGTTTCATGACCT-3' and $5^{\prime}$-CCCTCACTCGCCCTTCTTG- $3^{\prime}$; and $\beta$-actin (internal control) $5^{\prime}$-CT CCATCCTGGCCTCGCTGT-3' and $5^{\prime}$-GCTGTCACCTTCACCGTTCC- $3^{\prime}$. The relative expression levels were calculated by the $2^{-} \Delta \Delta C$ t method. The mRNA levels of each target gene were normalized to the levels of $\beta$-actin.

Western blotting. HEMs and HEKs were incubated under the indicated conditions were lysed and total protein was extracted from the tissue specimens and primary cells by the RIPA buffer, separated by $10 \%$ SDS-PAGE, and electrotransferred onto a polyvinylidene difluoride membrane (Millipore, Billerica, MA, USA). After blocking non-specific antibody binding by incubating the membrane in Tris-buffered saline containing $0.1 \%$ Tween- 20 and $5 \%$ nonfat dried milk for $2 \mathrm{~h}$ at room temperature, the membranes were incubated at $4{ }^{\circ} \mathrm{C}$ overnight with the following primary antibodies: from Abcam (Cambridge, MA, USA): anti-RNaseT2 (catalog no.: ab107313; 1:300 dilution), anti-Mitf (ab59232; 1:1000), anti-Tyr (ab61294; 1: 1000), anti-Trp-1 (ab83774; 1: 1000), and anti-Pomc (ab88976; 
1:1000); from Cell signaling (Danvers, MA, USA) anti-TRAF2 (4712P; 1:1000), anti-caspase3 (9662; 1:1000), anti-cleaved-caspase3 (9664; 1: 1000), and anti-caspase8 (9746; 1: 1000); or from Zen BioScience (Chengdu, Sichuan, China): anti- $\beta$-actin (8F10-G10; $1: 1000)$. Afterwards, the membranes were incubated with the appropriate horseradish peroxidase (HRP)-conjugated secondary antibody for $1 \mathrm{~h}$ at room temperature. Immunoreactive bands were detected by the Enhanced Chemiluminescence substrate (Pierce, Rockford, IL, USA) and visualized on the LAS-3000 Luminescence Image Analyzer (Fujjilm, Tokyo, Japan).

Immunofluorescent staining. HEK cells and HEM cells $\left(1 \times 10^{6}\right.$ per well) were seeded in triplicate into 6-well plates (Corning-Costar, Corning, NY, USA) onto gelatin-coated chamber slides and cultured for $24 \mathrm{~h}$, and then exposed to excessive NB-UVB and treated with different concentrations $\mathrm{H}_{2} \mathrm{O}_{2}$ and LPS for $48 \mathrm{~h}$. Next, the cultured primary HEMs and HEKs were fixed in $4 \%$ paraformaldehyde, permeabilized in a solution of $0.2 \%$ Triton X-100 and $2 \%$ bovine serum albumin (BSA) for $30 \mathrm{~min}$ at room temperature, and incubated with the anti- RNaseT2 antibody $(1: 100)$ at $4{ }^{\circ} \mathrm{C}$ overnight. Afterwards, the cells were incubated with the appropriate Cy3-conjugated secondary antibody (Jackson ImmunoResearch, West Grove, PA, USA; $1: 100$ ) at $37^{\circ} \mathrm{C}$ for $1 \mathrm{~h}$. Nuclei counterstaining was carried out by subsequent incubation with DAPI (BML-AP4020010; Biomol LP, Hong Kong, China). Next, the slides were mounted and observed under fluorescence microscope (Nikon, Tokyo, Japan).

Immunohistochemistry. The paraffin-embedded tissue sections $(5 \mu \mathrm{m}$ thickness) were deparaffinized, rehydrated, and subjected to antigen retrieval by boiling at $95^{\circ} \mathrm{C}$ in $10 \mathrm{mM}$ citrate buffer. After blocking of nonspecific antibody binding by incubating with $5 \% \mathrm{BSA}$, the sections were incubated with the antiRNaseT2 $(1: 50)$ at $4{ }^{\circ} \mathrm{C}$ overnight and the appropriate HRP-conjugated secondary antibody at $37^{\circ} \mathrm{C}$ for $1.5 \mathrm{~h}$. Following development by the 3.3diaminobenzidine chromogen substrate (Gene Tech, Shanghai, China), the sections were counterstained with hematoxylin and evaluated by microscopy. Negative technical controls were generated by replacing the primary antibody with phosphate-buffered saline alone.

Cell viability assay. At day 3 post transfection, HEK cells and HEM cells $\left(2 \times 10^{3}\right.$ per well) were collected and seeded in triplicate into 96-well plates in $100 \mu \mathrm{l}$ of growth medium. Different seeding densities were optimized at the beginning of the experiments (data not shown). The cell viability of the cultured primary HEMs and HEKs was assessed by using the Cell Count Kit-8 assay (Dojindo Laboratories, Kumamoto, Japan) according to the manufacturer's instructions at 24, 48, 72, 96, and $120 \mathrm{~h}$, respectively. Next, the absorbance of each well was obtained using a Dynatech MR5000 plate (Dynatech Laboratories, Alexandria, VA, USA) reader at a test wavelength of $490 \mathrm{~nm}$ with a reference wavelength of $630 \mathrm{~nm}$.

Apoptosis analysis. At day 3 post transfection, HEK cells and HEM cells $\left(1 \times 10^{6}\right.$ per well) were collected and seeded in triplicate into six-well plates in $2 \mathrm{ml}$ of growth medium and incubated in the presence or absence of the pancaspase inhibitor $10 \mu \mathrm{M} z V A D$-fmk (Sigma) for $24 \mathrm{~h}$. For oxidative stress assay, the medium was removed and the cells were incubated with increasing concentrations of $\mathrm{H}_{2} \mathrm{O}_{2}$ for $24 \mathrm{~h}$. The apoptotic nature of the cultured primary HEMs and HEKs was assessed at day 3 after seeding by using the Allophycocyanin-Conjugated Annexin-V/7-Amino-Actinomycin D kit (eBioscience, San Diego, CA, USA) and a flow cytometer equipped with the accompanying CellQuest data acquisition software (BD Biosciences, Franklin Lakes, NJ, USA).

Melanin quantification assay. Collected HEMs and HEKs were lysed by incubating in $20 \mathrm{mM}$ Tris and $0.1 \%$ Triton X-100 (pH 7.5) for 30 min on ice. After centrifugation, the pellets were subjected to two sequential wash steps of $5 \%$ trichloroacetic acid, a cold mixture of ethanol and ethyl ether (3:1), and cold ethyl ether. The resultant dried pellets were then dissolved in $1 \mathrm{mmol} / \mathrm{I} \mathrm{NaOH}$ by boiling for $10 \mathrm{~min}$, and measured by spectrophotometry at a wavelength of $490 \mathrm{~nm}$ (Thermo Scientific, Rockford, IL, USA). The concentration of melanin in each sample was calculated from the standard curves generated with synthetic melanin.

NB-UVB radiation and TYR activity assay. The NB-UVB radiation and TYR activity assay were performed as previously described. ${ }^{25}$

Lentiviral transduction. Wild-type human RNASET2 cDNA (NM_003730; Open-Biosystems, Thermo Scientific) was amplified by PCR (812 bp), confirmed by sequencing, inserted into the Agel and BamH sites of the GV287 green fluorescent protein-expressing lentiviral vector (GeneChem, Shanghai, China), and co-transfected into 293T cells along with helper constructs to produce lentivirus particles (range of titer yields: $2 \sim 5 \times 10^{7} \mathrm{TU} / \mathrm{ml}$ ) for transduction (at $30 \mathrm{MOI}$ ) into HEMs and HEKs for overexpression (OE) of RNASET2. RNASET2-ci was generated by the same procedure, except that the wild-type CDNA was triplemutated at residues 65,113 , and 118 (histidine $\rightarrow$ phenylalanine conversions) using the QuikChange Mutatgenesis Kit (Agilent Technologies, Santa Clara, CA, USA). Similarly, a TRAF2-binding-inhibited mutant (designated as RNASET2-ti) was generated by mutating residues 222-225 in the TRAF2-binding motif of the wild-type CDNA (PKQE $\rightarrow$ quadruple histidine conversion). HEMs and HEKs transfected with the empty lentiviral vector (designated as VECTOR) were used as controls. Expression of the RNASET2 wild-type and mutants was confirmed by western blot analysis.

Colony formation assay. Transfected HEK cells were collected and seeded into 6-well plates at 3000 cells/well and cultured for an additional 2 weeks. Dead cells (floaters) were removed by washing and the surviving cells (attached) were fixed in $30 \%$ ethanol, stained with $2 \%$ crystal violet, and observed under light microscope (Nikon). Each vector was assessed in triplicate.

Co-immunoprecipitation assay. The $\mathrm{C} 1$ plasmid vector expressing wildtype TRAF2 with a monomeric red fluorescent protein tag (designated as mRFP C1-TRAF2) was kindly gifted by Dr Qiu Junkang (Zen BioScience). A plasmid vector expressing wild-type RNASET2 with a FLAG tag (designated as FLAGRNASET2) was constructed by standard cloning. H1299 cells were grown to $60 \sim 80 \%$ confluency in 60-mm tissue culture dishes and co-transfected with $1.5 \mu \mathrm{g}$ of the mRFP C1-TRAF2 plasmid DNA and $1.5 \mu \mathrm{g}$ of the FLAG-RNASET2 plasmid DNA using the Lipofectamine LTX Plus reagent (Invitrogen). Immunoprecipitation and western blotting were performed as described previously. ${ }^{26}$

Statistical analysis. Data are expressed as mean \pm S.D. Student's $t$-test was used to determine significance of between-group differences. The doseresponse analyses were conducted by one-way ANOVA with Dunnett's multiple comparison test. A $P$-value of $<0.05$ was considered significant and a $P$-value of $<0.01$ was considered very significant. All statistical analyses and graphical representations of the data were performed by the GraphPad Prism (GraphPad Software Inc, San Diego, CA, USA).

\section{Conflict of Interest}

The authors declare no conflict of interest.

Acknowledgements. We thank Ming Guan, Bobin Chen, Boheng Zhang, Huimin Ren, Fang Huang, and Junkang Qiu for comments and suggestions concerning this manuscript. The project was financially supported by the Grant from Natural Science Foundation of China (Grant No. 81171519) and Shanghai Municipal Health Bureau (Grant No. XBR2011055).

1. Taieb A, Picardo M. Clinical practice. Vitiligo. N Engl J Med 2009; 360: 160-169.

2. Schaffer JV, Bolognia JL. The treatment of hypopigmentation in children. Clin Dermatol 2003; 21: 296-310.

3. Westerhof W, D'Ischia M. Vitiligo puzzle: the pieces fall in place. Pigment Cell Res 2007; 20: 345-359.

4. Quan C, Ren $Y Q$, Xiang LH, Sun LD, Xu AE, Gao XH et al. Genome-wide association study for vitiligo identifies susceptibility loci at 6q27 and the MHC. Nat Genet 2010; 42: 614-618.

5. Acquati F, Nucci C, Bianchi MG, Gorletta T, Taramelli R. Molecular cloning, tissue distribution, and chromosomal localization of the human homolog of the R2/Th/Stylar ribonuclease gene family. Methods Mol Biol 2001; 160: 87-101.

6. Acquati F, Possati L, Ferrante L, Campomenosi P, Talevi S, Bardelli S et al. Tumor and metastasis suppression by the human RNASET2 gene. Int J Oncol 2005; 26: 1159-1168.

7. Smirnoff $P$, Roiz L, Angelkovitch B, Schwartz B, Shoseyov O. A recombinant human RNASET2 glycoprotein with antitumorigenic and antiangiogenic characteristics: expression, purification, and characterization. Cancer 2006; 107: 2760-2769.

8. MacIntosh GC, Bariola PA, Newbigin E, Green PJ. Characterization of Rny1, the Saccharomyces cerevisiae member of the T2 RNase family of RNases: unexpected functions for ancient enzymes? Proc Natl Acad Sci USA 2001; 98: 1018-1023.

9. Thompson DM, Parker R. The RNase Rny1p cleaves tRNAs and promotes cell death during oxidative stress in Saccharomyces cerevisiae. J Cell Biol 2009; 185: 43-50. 
10. Thorn A, Steinfeld $R$, Ziegenbein $M$, Grapp $M$, Hsiao $H H$, Urlaub $H$ et al. Structure and activity of the only human RNase T2. Nucleic Acids Res 2012; 40: 8733-8742.

11. Gonzalvez F, Lawrence D, Yang B, Yee S, Pitti R, Marsters S et al. TRAF2 sets a threshold for extrinsic apoptosis by tagging caspase-8 with a ubiquitin shutoff timer. Mol Cell 2012 48: 888-899.

12. Huang CL, Nordlund JJ, Boissy R. Vitiligo: a manifestation of apoptosis? Am J Clin Dermatol 2002; 3: 301-308.

13. Thompson DM, Lu C, Green PJ, Parker R. tRNA cleavage is a conserved response to oxidative stress in eukaryotes. RNA 2008; 14: 2095-2103.

14. Acquati $\mathrm{F}$, Bertilaccio S, Grimaldi A, Monti L, Cinquetti $\mathrm{R}$, Bonetti $\mathrm{P}$ et al. Microenvironmental control of malignancy exerted by RNASET2, a widely conserved extracellular RNase. Proc Natl Acad Sci USA 2011; 108: 1104-1109.

15. Luhtala N, Parker R. T2 Family ribonucleases: ancient enzymes with diverse roles. Trends Biochem Sci 2010; 35: 253-259.

16. Slominski A, Wortsman J, Luger T, Paus R, Solomon S. Corticotropin releasing hormone and proopiomelanocortin involvement in the cutaneous response to stress. Physiol Rev 2000; 80: 979-1020

17. Bohm M, Wolff I, Scholzen TE, Robinson SJ, Healy E, Luger TA et al. alpha-Melanocytestimulating hormone protects from ultraviolet radiation-induced apoptosis and DNA damage. J Biol Chem 2005; 280: 5795-5802.

18. Luger TA, Scholzen TE, Brzoska T, Böhm M. New insights into the functions of alpha-MSH and related peptides in the immune system. Ann N Y Acad Sci 2003; 994 133-140.

19. Kadekaro AL, Kavanagh $\mathrm{R}$, Kanto $\mathrm{H}$, Terzieva $\mathrm{S}$, Hauser J, Kobayashi $\mathrm{N}$ et al. alpha-Melanocortin and endothelin-1 activate antiapoptotic pathways and reduce DNA damage in human melanocytes. Cancer Res 2005; 65: 4292-4299.
20. Francis $\mathrm{E}$, Wang $\mathrm{N}$, Parag $\mathrm{H}$, Halaban $\mathrm{R}$, Hebert DN. Tyrosinase maturation and oligomerization in the endoplasmic reticulum require a melanocyte-specific factor. J Biol Chem 2003; 278: 25607-25617.

21. Cao C, Healey S, Amaral A, Lee-Couture A, Wan S, Kouttab N et al. ATP-sensitive potassium channel: a novel target for protection against UV-induced human skin cell damage. J Cell Physiol 2007; 212: 252-263.

22. Steinfelder S, Andersen JF, Cannons JL, Feng CG, Joshi M, Dwyer D et al. The major component in schistosome eggs responsible for conditioning dendritic cells for Th2 polarization is a T2 ribonuclease (omega-1). J Exp Med 2009; 206: 1681-1690.

23. Everts B, Perona-Wright G, Smits HH, Hokke CH, van der Ham AJ, Fitzsimmons CM et al. Omega-1, a glycoprotein secreted by Schistosoma mansoni eggs, drives Th2 responses. J Exp Med 2009; 206: 1673-1680.

24. Yu N, Zhang S, Zuo F, Kang K, Guan M, Xiang L. Cultured human melanocytes express functional toll-like receptors 2-4, 7 and 9. J Dermatol Sci 2009; 56: 113-120.

25. Dong $D$, Jiang $M, X u X$, Guan $M, W u$ J, Chen $Q$ et al. The effects of NB-UVB on the hair follicle-derived neural crest stem cells differentiating into melanocyte lineage in vitro. J Dermatol Sci 2012; 66: 20-28.

26. He W, Zhao Y, Zhang C, An L, Hu Z, Liu Y et al. Rad9 plays an important role in DNA mismatch repair through physical interaction with MLH1. Nucleic Acids Res 2008; 36: 6406-6417.

cc) (-) $\Theta$ Cell Death and Disease is an open-access journal published by Nature Publishing Group. This work is licensed under a Creative Commons Attribution-NonCommercialNoDerivs 3.0 Unported License. To view a copy of this license, visit http://creativecommons.org/licenses/by-nc-nd/3.0/ 